\title{
Visual Perceptual Training Combined with Left Hand Somatosensory Stimulation for Reading in Acquired Right Brain Damage: A Pilot Study
}

\author{
Prabhjot Kaur Gambhir ${ }^{1 *}$ and Anuradha V Pai ${ }^{2}$ \\ ${ }^{1}$ Max Super Specialty Hospital, Saket, New Delhi, India \\ ${ }^{2}$ Department of Occupational Therapy, LTMMC and Government Hospital, Sion, Mumbai, India
}

\begin{abstract}
Background: Evaluation and treatment of perceptual dysfunction have traditionally been emphasized by occupational therapists working with adults with brain injury. Visual perceptual problems have been well documented as they have an impact on activities such as driving and reading. Limited information is available on the effectiveness
\end{abstract} of treatment.

Objective: To study the effect of visual perceptual training combined with left hand somatosensory stimulation on reading skills in individuals with acquired right brain damage

Methods: 10 subjects with acquired right brain damage were included in the study based on selection criteria. They underwent a comprehensive occupational therapy program of visual perceptual training emphasizing on reading- related tasks and left hand somatosensory stimulation for 6 weeks. Baseline, post intervention and followup assessments were done using Test of Visual Perceptual Skills-3 (TVPS-3), Letter Cancellation Test, and Reading Ability Screening Test (RAST).

Results: The program had a significant effect on reading skills with retention on follow-up as shown by RAST $(p<0.05)$. However, there was no significant improvement on TVPS-3 post intervention indicating a lack of generalization to skills other than reading.

Conclusion: Visual perceptual training and left hand somatosensory stimulation may be effective in remediation of reading related perceptual deficits in individuals with acquired right brain damage.

Keywords: Reading; Visual-perception; Somatosensory stimulation; Right brain damage

\section{Introduction}

Perception refers to the integration of sensory impressions into psychologically meaningful information. Visual perception is a process used by the central nervous system to adapt to the environment. It is the ability to select those stimuli that require attention and action, to integrate those stimuli with each other and prior information, and finally to interpret them [1].

Brain function lateralization is evident in the phenomena of right- or left-handedness. Left hemisphere directs attention towards the right half of the body space, whereas the right hemisphere directs attention towards both the right and left halves of the body space [2]. Right prefrontal, frontal and parietal regions [3]; right hippocampus [3], and right occipital region [4] are associated with visual perception in normal individuals as was studied by Giorgio Ganis et al. in their study on brain areas underlying visual perception based on fMRI.

Acquired Brain Injury (ABI) is an overarching term applied to describe insults to the brain that are not congenital or perinatal in nature. Usually the term $\mathrm{ABI}$ is used to describe the outcome of a distinct traumatic injury or single event pathology, but is not applied to the results of progressive disorders or degenerative diseases [5].

Reading is a complex cognitive process of decoding symbols for the intention of deriving meaning (reading comprehension) and/or constructing meaning [6]. Difficulty in reading, knows as acquired dyslexia or alexia, may occur with or without other language challenges, even when writing ability is intact. The inability or difficulty to read interferes with work and recreation for many survivors, making it difficult to follow written instructions. The ease and pleasure of reading is often replaced by effort and frustration.
Domains of visual perception that may affect reading are visual scanning, visual discrimination, and visual spatial relations. The problems encountered may include missing words or numbers, skipping individual words in a line, skipping lines, losing space on a page, inability to discriminate letters in foreground from background, combination of letters into words etc.

Visual inattention or neglect is usually associated with right cerebral hemisphere lesions which are attributed to brain function lateralization $[7,8]$. It is manifested by a failure to respond or orient to stimuli presented contra lateral to a brain lesion. Functionally, tasks such as reading or eating prove more difficult for individuals with right brain damage [9].

The remediation of reading deficits following acquired right brain damage was studied by various researchers. Evidence supports the remediation of acquired dyslexia with visual scanning training [10-12], and visual discrimination training [13] related to words and sentences. Further evidence is available regarding the activation of right side of the brain following remediation of reading problems [3]. Such activation has been observed with limb activation training, which involves somatosensory stimulation of the contralesional extremity

*Corresponding author: Prabhjot Kaur Gambhir, Max Super Specialty Hospital Saket, A-168, Sector-19, Noida, Distt. G B Nagar, New Delhi- 110017, India; Tel: 9971884421; E-mail: prabhjot.bkp@gmail.com

Received January 8, 2015; Accepted January 25, 2015; Published February 05, 2015

Citation: Gambhir PK, Pai AV (2015) Visual Perceptual Training Combined with Left Hand Somatosensory Stimulation for Reading in Acquired Right Brain Damage: A Pilot Study. Int J Neurorehabilitation 2: 147. doi:10.4172/2376-0281.1000147

Copyright: (c) 2015 Gambhir PK, et al. This is an open-access article distributed under the terms of the Creative Commons Attribution License, which permits unrestricted use, distribution, and reproduction in any medium, provided the original author and source are credited. 
[11]. However, no research has been conducted in India for remediation of reading deficits following brain injury.

Hence, effort was being made in this study to develop a remedial approach and verify its results scientifically by using a combined approach of visual perceptual training and left hand somatosensory stimulation for remediation of reading problems in individuals with acquired right brain damage.

\section{Materials and Methods}

In this prospective experimental study, 99 individuals with acquired right brain damage were screened. Out of these, 15 had associated cognitive problems, 9 had aphasia, and 63 were found to have no significant perceptual problems interfering with reading ability. 12 subjects matched the inclusion criteria out of which 10 subjects continued it through the entire process. Among them, 9 had stroke and one had suffered a traumatic brain injury. 3 of the subjects had hyperopia corrected by convex lenses. Informed consent was obtained and the participants were evaluated thrice during the study period- baseline, post intervention (after 6 weeks), and follow-up (2 weeks after completion of intervention to document retention effect).

\section{Inclusion criteria}

1. Unilateral acquired right brain damage ( confirmed with MRI)

2. Both sexes

3. Literate subjects (completed basic schooling 10+2)

4. At least 6 months post brain damage

5. Mini mental state examination (MMSE) score $>24$

6. Presence of visual perceptual disorders affecting reading ability

\section{Exclusion criteria}

1. Presence of aphasia

\begin{tabular}{|c|c|}
\hline No. of Cases & 10 \\
\hline $\begin{array}{l}\text { Mean } \\
\text { SD } \\
\text { Range }\end{array}$ & $\begin{array}{c}49.90 \\
12.31 \\
32-70 \text { yrs }\end{array}$ \\
\hline $\begin{array}{l}\text { Male } \\
\text { Female }\end{array}$ & $\begin{array}{l}07(70.0) \\
03(30.0)\end{array}$ \\
\hline $\begin{array}{r}\text { Time since ABI (months) } \\
\text { Mean } \\
\text { SD }\end{array}$ & $\begin{array}{l}15.80 \\
16.61\end{array}$ \\
\hline MMSE scores & $\begin{array}{l}26.20 \\
01.75\end{array}$ \\
\hline
\end{tabular}

(ABI- acquired brain injury; MMSE- Mini-Mental State Examination) Table 1: The demographic characteristics of the subjects
2. Presence of other neurological deficits affecting perceptual abilities

\section{Outcome measures}

1. Test of visual perceptual skills-3 (TVPS-3): it was used to determine visual perceptual strengths and weaknesses. The established norms for 18 year-olds were used to evaluate subjects (Martin’06) [14]

2. Letter cancellation test (LCT): was used to assess unilateral neglect (12)

3. Reading ability screening test (RAST): it is first subtest of the York Adult Assessment Battery- Revised with components of reading comprehension, accuracy, time, and rate) [15]

\section{Protocol}

All 10 subjects underwent comprehensive occupational therapy program for 1 hour, thrice a week for 6 weeks comprising of visual scanning, visual discrimination, and visual spatial training with reading-related tasks. Left hand somatosensory stimulation was incorporated with tactile stimulation using textures, identification of 3-D alphabets, and graphesthesia (writing single numbers or letters on dorsum of left hand) [1,11]. Baseline, post-intervention and follow up assessments were performed using TVPS-3, LCT, and RAST.

Statistical analysis was done using SPSS for Windows, version 13.0. Mean value of the total score was calculated and paired T-test was used for the comparison of pre intervention - post intervention as well as pre intervention - follow up TVPS-3, LCT, and RAST scores. $P$ value less than or equal to 0.05 was considered significant.

\section{Results}

Table 1 shows the demographic characteristics of the subjects. The age of subjects ranged from 32 to 70 years with an average age of 49.90 years. 7 subjects were males and 3 were females. Average time since acquired brain injury was 15.80 months. Mean score of MMSE was 26.20 .

Table 2 shows that the mean TVPS-3 score was 64.90 at baseline. After the treatment, no statistically significant change was observed in mean TVPS-3 from baseline. At the follow-up, mean TVPS-3 scores increased significantly to $49.2 \%$ from baseline (Figure 1). The mean LCT was 24.00 pre-intervention. Post intervention, mean LCT showed an increase of $39.6 \%$ from baseline where the change was significant. No change was observed in mean LCT at the end of follow up from baseline (Figure 1). The mean RAST was 12.10 preintervention. At post and follow up period, mean RAST increased by $67.8 \%$ respectively from baseline where the change was statistically significant (Figure 1).

\begin{tabular}{|c|c|c|c|}
\hline Duration & Mean TVPS $\left(\bar{X}_{ \pm} S D\right)(N=10)$ & Mean LCT $\left(\bar{X}_{ \pm} S D\right)(N=10)$ & Mean RAST $\left(\bar{X}_{ \pm} S D\right)(N=10)$ \\
\hline Pre intervention (pre) & $64.90 \pm 08.31$ & $24.00 \pm 6.15$ & $12.10 \pm 3.35$ \\
\hline Post intervention (post) & $78.20 \pm 24.32$ & $33.50 \pm 2.46$ & $20.30 \pm 2.06$ \\
\hline Follow-up (F/U) & $96.80 \pm 13.32$ & $26.00 \pm 5.94$ & $20.30 \pm 2.16$ \\
\hline Difference (Pre - Post $)(p$ value) & $13.30 \pm 29.99(0.1943)$ & ${ }^{*} 9.50 \pm 4.79(0.0001)$ & ${ }^{*} 8.20 \pm 1.93(0.0000)$ \\
\hline Difference $($ Pre $-F / U)(p$ value $)$ & $* 31.90 \pm 12.58(0.0000)$ & $2.00 \pm 3.46(0.1008)$ & $* 8.20 \pm 3.12(0.0000)$ \\
\hline
\end{tabular}

By Student 't' Test * Significant

(TVPS-Test of Visual Perceptual Skills, LCT- Letter Cancellation Test, RAST- Reading Ability Screening Test)

Table 2: Changes in mean scores of TVPS-3, LCT, and RAST. 


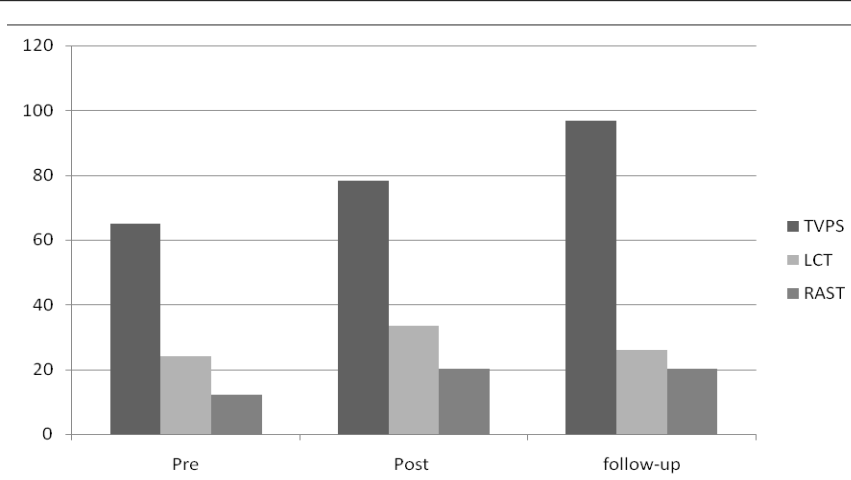

(TVPS- Test of Visual Perceptual Skills-3; LCT- Letter Cancellation Test; RASTReading ability Screening Test)

Figure 1: The changes in mean scores of TVPS-3, LCT, and RAST at preintervention, post-intervention, and follow-up.

\section{Discussion}

Visual perceptual skills related to reading improved in subjects following 6 weeks of comprehensive occupational therapy program.

Visual scanning may aid in treatment of hemineglect rehabilitation related to reading skills [11]. The subjects were able to scan the reading material on receiving visual scanning training though literature supports the effects being restricted to the task which was specifically trained, which was reading in the current study [12].

Visual discrimination training may aid the subjects in discriminating letters, thereby, emphasizing the positioning of these letters to form words and, finally, reading of sentences. This may enhance the ability of the individuals to synthesize reading material as a whole and utilize reading skills in everyday life. Similar training effects were shown to improve reading skills, specifically reading words, by King et al. [13].

Visual spatial training has been shown to reduce spatial hemineglect in right brain damage patients by Piccardi et al. [16], though its effectiveness in remediating reading skills has never been emphasized. The comprehensive occupational therapy (OT) program comprised visual spatial training emphasizing tracking, anchoring techniques etc to improve reading ability of the subjects.

Following 6 weeks of intervention, all the subjects showed an improvement on standard TVPS scores but they were not statistically significant ( $\mathrm{p}=0.1943$ ) (Table 2). These findings could be attributed to the reason that there is erratic and inconsistent generalization of training effects to non- trained tasks as the treatment program in current study pertained to reading- related tasks only [17]. However, after 2 weeks of follow-up, which was the non-intervention period, the results from baseline were significant $(\mathrm{p}<0.05)$ owing to the practice effect of the test observed after 2 weeks.

Post intervention, there was a statistically significant improvement on LCT scores $(\mathrm{p}<0.05)$ as the training program comprised scanning training involving reading related skills (Table 2). Such training improves visual exploration of extra personal space as was postulated by Polanowska et al. and studied using letter cancellation tests along with letter reading task [11]. But the retention effect of these scores in the follow-up evaluation was absent $(\mathrm{p}=0.1008)$.

Yvonne Goddard emphasized the need to develop a remedial program addressing the needs of brain injury patients for reading skills [18]. Such a program was developed in this study and assessment of reading skills was done using RAST. The comprehensive OT program was found to be effective in improving reading skills as was evident by statistically significant $(\mathrm{p}<0.05)$ results both post-intervention and at the time of follow-up (Table 2).

Large somatosensory fields for the hands are localized to the parietal region. The somatosensory stimulation provided in this study might have contributed to an increase in the activation level in this area, which is crucial for spatial attention. Polanowska et al. concluded in their study, combining the effects of left hand somatosensory stimulation with visual scanning, that the combined approach was more effective for rehabilitation of stroke patients [11]. This signifies the role of left hand somatosensory stimulation in improving reading skills in right acquired brain damage individuals.

The study devised a comprehensive occupational therapy program including visual perceptual training combined with left hand somatosensory stimulation which may assist in remediating perceptual deficits associated with reading in individuals with acquired right brain damage. However, no generalization of effects was observed to tasks other than related to reading.

The study may be undertaken with a larger sample size including a control group for better generalization of results. The duration of intervention may be increased in order to have good retention effect. To establish it as a part of permanent improvement, the study of retention effect may be done at least after 3-6 months.

\section{Acknowledgement}

We sincerely thank Pratibha $\vee$ Rege, Ex-Head of Occupational Therapy department at All India Institute of Physical Medicine and Rehabilitation, Mumbai (India) and all the participants in the study for their support throughout the course of the study.

\section{References}

1. Robertson IH, Heutink J (2002) Rehabilitation of Unilateral Neglect. In: W.H. Brouwer et al. (Eds.) Neuropsychological Rehabilitation: a Cognitive Approach. Amsterdam

2. Heilman KM, Van Den Abell T (1980) Right hemisphere dominance for attention: The mechanisms underlying hemispheric asymmetries of inattention neglect. Neurology. 30: 327-330.

3. Noppeney U, Price CJ, Duncan JS, Koepp MJ (2004) Reading Skills after Left Anterior Temporal Lobe Resection: An fMRI Study. Journal of Neuropsychology. 5: 55-67.

4. Ganis G, Thompson WL, Kosslyn SM (2004) Brain areas underlying visual mental imagery and visual perception: an fMRI study. Cognitive Brain Research. 20: 226-241.

5. Stokes M (2004) Physical Management in Neurological Rehabilitation. Elsevier limited. $2^{\text {nd }}$ edition

6. Klein RM, McMullen P (2001) Converging Methods for Understanding Reading and Dyslexia. Massachusetts institute of Technology. $2^{\text {nd }}$ edition

7. Pedretti LW, Pendleton HM, Schultz-Krohn W (2013) Occupational Therapy Practice Skills for Physical Dysfunction. Elsevier limited. $7^{\text {th }}$ edition

8. Spiers PA, Schomer DL, Blume HW (1990) Visual neglect during intracarotid amobarbital testing. Neurology. 40: 1600-1606.

9. Trombly CA, Radomski MV (2008) Occupational Therapy for Physical Dysfunction. Lippincott Williams and Wilkins. $6^{\text {th }}$ edition.

10. Weinberg J, Diller L, Gordon WA, Gerstman LJ, Lieberman A, et al. (1977) Visual Scanning Training Effect on Reading-Related Tasks in Acquired Right Brain Damage. Archives of Physical Medicine and Rehabilitation. 58: 479-486.

11. Polanowska K, Seniow J, Paprot E, Lesniak M, Czlonkowska A (2009) Left Hand Somatosensory Stimulation combined with Visual Scanning Training in Rehabilitation of Post-Stroke Hemineglect: A Randomized Double-Blind Study. Neuropsychological Rehabilitation. 19: 364-382. 
Citation: Gambhir PK, Pai AV (2015) Visual Perceptual Training Combined with Left Hand Somatosensory Stimulation for Reading in Acquired Right Brain Damage: A Pilot Study. Int J Neurorehabilitation 2: 147. doi:10.4172/2376-0281.1000147

12. Wagenaar RC, Wieringen $P$, Netelenbos JB, Meijer OG, Kulk DJ (1992) Transfer of Scanning Training Effects in Visual Inattention after Stroke: Five Singe Case Studies. Disability and Rehabilitation. 14: 51-60.

13. King EM (1964) Effects of Different Kinds of Visual Discrimination Training on Learning to Read Words. Journal of Educational Psychology. 55: 325-333.

14. Brown T, Mullins E, Stagnitti K (2009) The Concurrent Validity of Three Visual Perception Tests used with Adults. Perceptual and Motor Skills. 109: 99-118.

15. Warmington M, Stothard SE, Snowling MJ (2012) Assessing Dyslexia in Higher Education: The York Adult Assessment Battery-Revised. Journal of Research in Special Educational Needs. 13: 48-56.
16. Piccardi L, Nico D, Bureca I, Matano A, Guariglia C (2006) Efficacy of VisuoSpatial Training in Right Brain Damage Patients with Spatial Hemineglect and Attention Disorders. Cortex. 42: 973-982.

17. Gouvier WD, Bua BG, Blanton PD, Urey JR (1987) Behavioural Changes following Visual Scanning Training: Observations of Five Cases; International Journal of Clinical Neuropsychology. 9: 74-80.

18. Goddard Y (2009) Using Basic Reading Skills Instruction and Formative Assessments to Teach an Adult with Traumatic Brain Injury to Read. Remedial and Special education. 30: 283-299. 